\title{
Importancia de la educación emocional en la formación inicial del profesorado
}

\author{
Pilar Mireya Rueda Carcelén, Gemma Filella Guiu \\ Universitat de Lleida \\ gfilella@pip.udl.cat
}

RESUM

Importància de l'educació emocional en la formació inicial del professorat

Aquest article presenta la importància de formar en competències emocionals als futurs docents de les diferents etapes educatives. L'Educació Emocional a tot ésser humà, i en especial als docents, permet donar resposta als nous reptes que planteja la societat del segle XXI: educar per a potenciar la capacitat crítica en una societat del coneixement, capacitat per tolerar la frustració, postergar la recompensa i immediatesa i, sobretot, guiar els futurs adults cap a la competència entesa com a adaptació, superació i realització intra i interpersonal, no com a rivalitat i poder.

Paraules Clau

Competència Emocional, Reptes del segle XXI, Formació inicial del docent.

\section{RESUMEN}

\section{Importancia de la educación emocional en la formación inicial del profesorado}

Este artículo presenta la importancia de formar en competencias emocionales a los futuros docentes de las diferentes etapas educativas. La Educación Emocional en todo ser humano, y en especial a los docentes, permite dar respuesta a los nuevos retos que plantea la sociedad del siglo XXI: educar para potenciar la capacidad crítica en una sociedad del conocimiento, capacidad para tolerar la frustración, postergar la recompensa e inmediatez y, sobre todo, guiar a los futuros adultos hacia la competencia entendida como adaptación, superación y realización intra e interpersonal y no como rivalidad y poder.

Palabras clave

Competencia Emocional, Retos del siglo XXI, Formación inicial del docente.

\section{RÉSUMÉ}

\section{Importance de l'éducation émotionnelle dans la formation des futurs enseignants}

Cet article présente l'importance de la formation des futurs enseignants des différents niveaux de l'enseignement aux compétences émotionnelles. L'éducation émotionnelle de tous les êtres humains et en particulier des enseignants peut apporter des réponses aux nouveaux défis du XXIe siècle: l'éducation pour améliorer la capacité critique dans une société de la connaissance, la capacité à tolérer la frustration, le retard dans les récompenses, le manque d'immédiateté et, surtout, un guide pour les futurs adultes dans cette compétence, entendue comme adaptation, amélioration et réalisation intra et interpersonnelle plutôt que comme rivalité et rapports de pouvoir. 


\section{Mots-Cles}

Compétence émotionnelle, Défis du XXIe siècle, Formation initiale des enseignants.

\section{AbSTRACT}

\section{The importance of emotional education in the future teachers' initial training.}

This paper discusses the importance of training in emotional competences the future teachers of the different educational stages. Emotional Education in every human being, especially in teachers, allows responding in a more proper way to the new challenges of the $21^{\text {st }}$ century: educating to enhance critical thinking in a society of knowledge and the ability to tolerate frustration, delaying the reward and immediacy and, above all, guiding future adults to competition understood as adaptation, improvement and both intra- and interpersonal fulfilment instead of rivalry and power.

\section{KEYWORDS}

Emotional Competence, Challenges of the $21^{\text {st }}$ Century, Teachers' initial training.

\section{Por qué es importante enseñar la Educación Emocional}

Las competencias emocionales son competencias básicas que facilitan un adecuado ajuste personal, social, académico y laboral. En este sentido, la educación emocional es un proceso educativo continuo y permanente, puesto que debe estar presente a lo largo de todo el currículum académico y en la formación permanente a lo largo de toda la vida (Bisquerra, 2000). Tiene un enfoque del ciclo vital. A lo largo de toda la vida se pueden producir conflictos que afectan al estado emocional y que requieren una atención psicopedagógica.

La educación emocional es una forma de prevención primaria inespecífica, consistente en intentar minimizar la vulnerabilidad a las disfunciones o prevenir su ocurrencia (Bisquerra, 2007). Los nińos y jóvenes necesitan, en su desarrollo hacia la vida adulta, que se les proporcionen recursos y estrategias para enfrentarse con las inevitables experiencias que la vida nos depara.

La inteligencia es una aptitud; el rendimiento es lo que uno consigue; la competencia indica en qué medida el rendimiento se ajusta a unos patrones determinados. De forma análoga se puede considerar que la inteligencia emocional es una capacidad (que incluye aptitud y habilidad); el rendimiento emocional representaría el aprendizaje. Se da competencia emocional cuando uno ha logrado un determinado nivel de rendimiento emocional (Mayer y Salovey, 1997; Saarni, 1988).

La formación en competencias emocionales es necesaria para que tanto los niños (futuros adultos) como el profesorado puedan adaptarse con éxito. Pero no es sólo importante de cara a poder desarrollar dichas competencias en los estudiantes o a prevenir desajuste en la salud mental del profesorado, sino también para crear entornos favorecedores de aprendizaje (Berrocal y Extremera, 2008).

Diversos investigadores de reconocido prestigio como Durlak, Weissberg, Dymnicki, Taylor \& Schellinger (2011), Zins, Payton \& O’ Brien (2007) etc., concluyen que los beneficios de la educación emocional se manifiestan principalmente en progresos significativos en el alumnado en cuanto a competencias sociales y emocionales, mejora de actitudes hacia uno mismo, hacia los demás y hacia la escuela, comportamiento positivo en clase, mejora del clima de clase, reducción del comportamiento disruptivo, mejora del rendimiento académico y disminución de problemas y conflictos, problemas de comportamiento, agresividad, violencia, ansiedad y estrés (Filella, G. 2014). 
La competencia emocional de los docentes es necesaria para su propio bienestar personal y para la efectividad y calidad a la hora de llevar a cabo los procesos de enseñanza-aprendizaje del aula, en general, y el desarrollo socio-emocional en los alumnos, en particular (Sutton y Wheatly, 2003).

Las competencias emocionales son las siguientes:

\section{Conciencia Emocional}

Podemos definir la conciencia emocional como la capacidad para tomar conciencia de las propias emociones y de las emociones de los demás, incluyendo la habilidad para captar el clima emocional de un contexto determinado. Dentro de este bloque se pueden especificar una serie de aspectos como los siguientes.

- Toma de conciencia de las propias emociones. Es la capacidad para percibir con precisión los propios sentimientos y emociones; identificarlos y etiquetarlos.

- Dar nombre a las emociones. Es la eficacia en el uso del vocabulario emocional adecuado y utilizar las expresiones disponibles en un contexto cultural determinado para designar los fenómenos emocionales.

- Comprensión de las emociones de los demás. Es la capacidad para percibir con precisión las emociones y sentimientos de los demás y de implicarse empáticamente en sus vivencias emocionales.

- Tomar conciencia de la interacción entre emoción, cognición y comportamiento. Los estados emocionales inciden en el comportamiento y éstos en la emoción. Emoción, cognición y comportamiento están en interacción continua, de tal forma que resulta difícil discernir cuál es primero.

\section{Regulación Emocional}

La regulación emocional es la capacidad para manejar las emociones de forma apropiada. Supone tomar conciencia de la relación entre emoción, cognición y comportamiento; tener buenas estrategias de afrontamiento; capacidad para autogenerarse emociones positivas, etc.

Las microcompetencias que la configuran son:

- Expresión emocional apropiada. Es la capacidad para expresar las emociones de forma apropiada. Implica la habilidad para comprender que el estado emocional interno no necesita corresponder con la expresión externa. Esto se refiere tanto en uno mismo como en los demás. En niveles de mayor madurez, supone la comprensión del impacto que la propia expresión emocional y el propio comportamiento, puedan tener en otras personas.

- Regulación de emociones y sentimientos. Es la regulación emocional propiamente dicha. Esto significa aceptar que los sentimientos y emociones a menudo deben ser regulados. Lo cual incluye: regulación de la impulsividad (ira, violencia, comportamientos de riesgo); tolerancia a la frustración para prevenir estados emocionales negativos (ira, estrés, ansiedad, depresión); perseverar en el logro de los objetivos a pesar de las dificultades; capacidad para diferir recompensas inmediatas a favor de otras más a largo plazo pero de orden superior, etc.

- Habilidades de afrontamiento: Habilidad para afrontar retos y situaciones de conflicto, con las emociones que generan. Esto implica estrategias de autorregulación para gestionar la intensidad y la duración de los estados emocionales. 
- Competencia para autogenerar emociones positivas. Es la capacidad para autogenerarse y experimentar de forma voluntaria y consciente emociones positivas (alegría, amor, humor, fluir) y disfrutar de la vida. Capacidad para auto-gestionar el propio bienestar emocional en busca de una mejor calidad de vida.

\section{Autonomía Emocional}

Es un concepto amplio que incluye un conjunto de características y elementos relacionados con la autogestión personal, entre las que se encuentran la autoestima, actitud positiva ante la vida, responsabilidad, capacidad para analizar críticamente las normas sociales, la capacidad para buscar ayuda y recursos, así como la autoeficacia emocional. Incluye las siguientes micro-competencias:

- Autoestima. se refiere a tener una imagen positiva de sí mismo; estar satisfecho de sí mismo; mantener buenas relaciones consigo mismo.

- Automotivación. Es la capacidad de automotivarse e implicarse emocionalmente en actividades diversas de la vida personal, social, profesional, de tiempo libre, etc. Motivación y emoción van de la mano. Automotivarse es esencial para dar un sentido a la vida.

- Autoeficacia emocional. Se refiere a la percepción de que "se es capaz" (eficaz) en las relaciones sociales y personales gracias a las competencias emocionales. El individuo se percibe a sí mismo con capacidad para sentirse como desea; para generarse las emociones que necesita.

- Responsabilidad. Se refiere a la capacidad para responder de los propios actos. Es la intención de implicarse en comportamientos seguros, saludables y éticos. Asumir la responsabilidad en la toma de decisiones. Ante la decisión de ¿qué actitudes (positivas o negativas) voy a adoptar ante la vida?, en virtud de la autonomía y libertad, decidir con responsabilidad, sabiendo que en general lo más efectivo es adoptar una actitud positiva.

- Actitud positiva. Es la capacidad para decidir que voy a adoptar una actitud positiva ante la vida. A pesar de que siempre van a sobrar motivos para que la actitud sea negativa. Saber que en situaciones extremas, lo heroico es adoptar una actitud positiva, aunque cueste. Siempre que sea posible manifestar optimismo y mantener actitudes de amabilidad y respeto a los demás. Por extensión, la actitud positiva repercute en la intención de ser bueno, justo, caritativo y compasivo.

- Análisis crítico de normas sociales. Es la capacidad para evaluar críticamente los mensajes sociales, culturales y de los mass media, relativos a normas sociales y comportamientos personales. Esto tiene sentido de cara a no adoptar los comportamientos estereotipados propios de la sociedad irreflexiva y acrítica. La autonomía debe ayudar a avanzar hacia una sociedad más consciente, libre, autónoma y responsable.

- Resiliencia. Es la capacidad que tiene una persona para enfrentarse con éxito a unas condiciones de vida sumamente adversas (pobreza, guerras, orfandad, etc.).

\section{La competencia social}

Es la capacidad para mantener buenas relaciones con otras personas. Esto implica dominar las habilidades sociales básicas, capacidad para la comunicación efectiva, respeto, actitudes prosociales, asertividad, etc. Las microcompetencias que incluye la competencia social son las siguientes.

- Dominar las habilidades sociales básicas. La primera de las habilidades sociales es escuchar. Sin ella, difícilmente se pueda pasar a las demás: saludar, despedirse, dar las gracias, pedir un favor, 
manifestar agradecimiento, pedir disculpas, aguardar turno, mantener una actitud dialogante, etc.

- Respeto por los demás. Es la intención de aceptar y apreciar las diferencias individuales y grupales y valorar los derechos de todas las personas. Esto se aplica en los diferentes puntos de vista que puedan surgir en una discusión.

- Practicar la comunicación receptiva. Es la capacidad para atender a los demás tanto en la comunicación verbal como no verbal para recibir los mensajes con precisión.

- Practicar la comunicación expresiva. Es la capacidad para iniciar y mantener conversaciones, expresar los propios pensamientos y sentimientos con claridad, tanto en comunicación verbal como no verbal, y demostrar a los demás que han sido bien comprendidos.

- Compartir emociones. Compartir emociones profundas no siempre es fácil. Implica la conciencia de que la estructura y naturaleza de las relaciones vienen en parte definidas tanto por el grado de inmediatez emocional, o sinceridad expresiva, como por el grado de reciprocidad o simetría en la relación.

- Comportamiento prosocial y cooperación. Es la capacidad para realizar acciones en favor de otras personas, sin que lo hayan solicitado. Aunque no coincide con el altruismo, tiene muchos elementos en común.

- Asertividad. Significa mantener un comportamiento equilibrado entre la agresividad y la pasividad. Esto implica la capacidad para defender y expresar los propios derechos, opiniones y sentimientos, al mismo tiempo que se respeta a los demás, con sus opiniones y derechos. Decir "no" claramente y mantenerlo y aceptar que el otro te pueda decir "no". Hacer frente a la presión de grupo y evitar situaciones en las cuales uno puede verse coaccionado para adoptar comportamientos de riesgo. En ciertas circunstancias de presión, procurar demorar la toma de decisiones y la actuación, hasta sentirse adecuadamente preparado, etc.

- Prevención y solución de conflictos. Es la capacidad para identificar, anticiparse o afrontar resolutivamente conflictos sociales y problemas interpersonales. Implica la capacidad para identificar situaciones que requieren una solución o decisión preventiva y evaluar riesgos, barreras y recursos. Cuando inevitablemente se producen los conflictos, afrontarlos de forma positiva, aportando soluciones informadas y constructivas. La capacidad de negociación y mediación son aspectos importantes de cara a una resolución pacífica del problema, considerando la perspectiva y los sentimientos de los demás.

- Capacidad para gestionar situaciones emocionales. Es la habilidad para reconducir situaciones emocionales en contextos sociales. Se trata de activar estrategias de regulación emocional colectiva. Esto se superpone con la capacidad para inducir o regular las emociones en los demás.

\section{Las competencias para la vida y el bienestar}

Son la capacidad para adoptar comportamientos apropiados y responsables para afrontar satisfactoriamente los desafíos diarios de la vida, ya sean personales, profesionales, familiares, sociales, de tiempo libre, etc. Las competencias para la vida permiten organizar nuestra vida de forma sana y equilibrada, facilitándonos experiencias de satisfacción o bienestar. Como microcompetencias se incluyen las siguientes.

- Fijar objetivos adaptativos. Es la capacidad para fijar objetivos positivos y realistas. Algunos a corto plazo (para un día, semana, mes); otros a largo plazo (un año, varios años). 
- Toma de decisiones. Desarrollar mecanismos personales para tomar decisiones sin dilación en situaciones personales, familiares, académicas, profesionales, sociales y de tiempo libre, que acontecen en la vida diaria. Supone asumir la responsabilidad por las propias decisiones, tomando en consideración aspectos éticos, sociales y de seguridad.

- Buscar ayuda y recursos. Es la capacidad para identificar la necesidad de apoyo y asistencia y saber acceder a los recursos disponibles apropiados.

- Ciudadanía activa, participativa, crítica, responsable y comprometida. Lo cual implica reconocimiento de los propios derechos y deberes; desarrollo de un sentimiento de pertenencia; participación efectiva en un sistema democrático; solidaridad y compromiso; ejercicio de valores cívicos; respeto por los valores multiculturales y la diversidad, etc. Esta ciudadanía se desarrolla a partir del contexto local, pero se abre a contextos más amplios (autonómico, estatal, europeo, internacional, global). Las competencias emocionales son esenciales en la educación para la ciudadanía (Bisquerra, 2008).

- Bienestar emocional. Es la capacidad para gozar de forma consciente de bienestar (emocional, subjetivo, personal, psicológico) y procurar transmitirlo a las personas con las que se interactúa. Adoptar una actitud favorable al bienestar. Aceptar el derecho y el deber de buscar el propio bienestar, ya que con ello se puede contribuir activamente al bienestar de la comunidad en la que uno vive (familia, amigos, sociedad).

- Fluir. Capacidad para generar experiencias óptimas en la vida profesional, personal y social.

Las aplicaciones de la educación emocional se pueden dejar sentir en múltiples situaciones: comunicación efectiva y afectiva, resolución de conflictos, toma de decisiones, prevención inespecífica (consumo de drogas, sida, violencia, anorexia, intentos de suicidio). En último término se trata de desarrollar la autoestima, con expectativas realistas sobre sí mismo, desarrollar la capacidad de fluir y la capacidad para adoptar una actitud positiva ante la vida. Todo ello de cara a posibilitar un mayor bienestar emocional, que redunda en un mayor bienestar social.

\section{Diferentes recursos para el proceso de enseñanza-aprendizaje de la Educación Emocional}

La educación de las competencias emocionales sigue una metodología eminentemente práctica (dinámica de grupos, autorreflexión, razón dialógica, juegos...). Para apoyar la labor del profesorado y de las familias se han elaborado materiales prácticos, con ejercicios y actividades enfocadas al desarrollo de la inteligencia emocional y las competencias emocionales. A continuación referenciamos algunos recursos didácticos.

Para la Etapa de Educación Infantil:

Gómez-Bruguera, J. (2003). Educación Emocional y lenguaje en la escuela. Barcelona: Octaedro-Rosa Sensat.

GROP (2009). Actividades para el desarrollo de la inteligencia emocional en los niños. Barcelona:

Parramón. (Versión en castellano, catalán, francés, portugués, holandés y esloveno).

López-Cassà, E. (coord.). (2003). Educación emocional. Programa para 3-6 años. Barcelona: Praxis. Palou-Vivens, S. (2004). Sentir y crecer. EL crecimiento emocional en la infancia. Barcelona: Graó. Shapiro, L.E. (1998). La inteligencia emocional de los niños. Barcelona: Ediciones B.

Para la Etapa de Educación Primaria:

Carpena, A. (2003). Educación socioemocional en la etapa de primaria. Barcelona: Octaedro. 
Filella, G. (2014). Aprendre a Conviure. Barcelona: Barcanova.

Renom, A. (coord.). (2003). Educación emocional. Programa para la educación primaria. Barcelona: Praxis.

Vallés-Arándiga, A. (1999). SICLE. Siento inteligente con las emociones. Valencia: Promolibro.

Para la Educación Secundaria Obligatoria (ESO)

Elias, M.J., Tobias, S.E., Friedlander, B.S. (2001). Educar adolescentes con inteligencia emocional. Barcelona: Plaza Janés.

Pascual, V., Cuadrado, M. (coords.) (2001). Educación emocional. Programa de actividades para la Educación Secundaria Obligatoria. Barcelona: Praxis.

Para la vida adulta:

Güell-Barceló, M., Muñoz-Redon, J. (2000). Desconócete a ti mismo. Programa de alfabetización emocional. Barcelona: Paidós.

Güell, M., Muñoz, J (coords.). (2003). Educación emocional. Programa para la educación secundaria postobligatoria. Barcelona: Praxis.

Redorta, J., Obiols, M., Bisquerra, R. (2006). Emoción y conflicto. Aprenda a manejar las emociones. Barcelona: Paidós.

Bisquerra Alzina, R. \& Pérez Escoda, N. (2007). "Las competencias emocionales”. Educación XX1: revista de la Facultad de Educación, no 10, p. 61-82. Madrid: UNED.

\section{Conclusión}

La formación en competencias emocionales y su didáctica es imprescindible para los estudiantes del grado de Magisterio en todos sus niveles. El estudio de las emociones no solamente va a permitir el conocimiento de uno mismo como ser humano en su desarrollo personal, sino también en el profesional. La clave de la educación no es únicamente transmitir, sino que también es ser un ejemplo a seguir. Un docente concebido como una persona que acompaña, guía, motiva, comprende, facilita, reta, ilusiona y crea el clima adecuado en su aula ( tanto con los nińos) y en el Centro Educativo (toda la comunidad educativa) para que fluya el aprendizaje tanto dentro como fuera de las aulas.

La formación inicial de los docentes en competencias emocionales es una necesidad de ajuste y adaptación a los nuevos retos que nos plantea el siglo XXI: capacidad de tolerar la frustración, postergar la recompensa e inmediatez, desarrollar una capacidad crítica capaz de discernir lo más apropiado según la circunstancia y contexto y sobre todo, guiar a los futuros adultos hacia la competencia entendida como adaptación, superación y realización intra e interpersonal y no como rivalidad y poder.

\section{Bibliografía}

BISQUERRA, R., Educación emocional y bienestar, Barcelona, Praxis, 2000.

BISQUERRA, R., Psicopedagogía de las emociones, Madrid, Ed. Síntesis, 2009.

CARPENA, A., Educación socioemocional en la etapa de primaria. Barcelona, Octaedro, 2003. 
DURLAK, J. A., WEISSBERG, R. P., DYMNICKI, A. B., TAYLOR, R. D., y SCHELLINGER, K. B. "The impact of enhancing students' social and emotional learning: A meta-analysis of school-based universal interventions", Child development, 82(1), 2011, p.405-432.

ELIAS, M.J., TOBIAS, S.E. y FRIEDLANDER, B.S., Educar adolescentes con inteligencia emocional, Barcelona, Plaza Janés, 2001.

FILELLA, G., Aprendre a Conviure, Barcelona, Barcanova, 2014.

GOLEMAN, D., La práctica de la inteligencia emocional, Barcelona, Kairós, 1999.

GÓMEZ-BRUGUERA, J., Educación Emocional y lenguaje en la escuela, Barcelona, Octaedro-Rosa Sensat, 2003.

GROP, Actividades para el desarrollo de la inteligencia emocional en los niños, Barcelona, Parramón, 2009. (Versión en castellano, catalán, francés, portugués, holandés y esloveno).

GÜELL, M. y MUÑOZ, J. (coords.), Educación emocional. Programa para la educación secundaria postobligatoria, Barcelona, Praxis, 2003.

GÜELL, M. y MUÑOZ, J., Desconócete a ti mismo. Programa de alfabetización emocional, Barcelona, Paidós, 2000.

LÓPEZ-CASSÀ, E. (coord.), Educación emocional. Programa para 3-6 años. Barcelona, Praxis, 2003. MAYER, J.D. y SALOVEY, P., Mayer-Salovery-Caruso Emotional Intelligence Test, Multi-Health Systems Incorporated, 2007.

MARTÍN, R.P., BERROCAL, P.F., y BRACKETT, M.A., "La inteligencia emocional como una competencia básica en la formación inicial de los docentes: algunas evidencias" Electronic journal of research in educational psychology, 6 (15), 2008, pp. 437-454.

PALOU-VIVENS, S., Sentir y crecer. EL crecimiento emocional en la infancia, Barcelona, Graó, 2004.

PASCUAL, V. y CUADRADO, M. (coords.), Educación emocional. Programa de actividades para la Educación Secundaria Obligatoria, Barcelona, Praxis, 2001.

REDORTA, J., OBIOLS, M. y BISQUERRA, R., Emoción y conflicto. Aprenda a manejar las emocione, Barcelona, Paidós, 2006.

RENOM, A. (coord.), Educación emocional. Programa para la educación primaria, Barcelona, Praxis, 2003.

SAARNI, C., "Children's understanding of the interpersonal consequences of dissemblance of nonverbal emotional-expressive behavior", Journal of Nonverbal Behavior, 12(4),1988, pp. $275-$ 294.

SHAPIRO, L.E., La inteligencia emocional de los niños, Barcelona, Ediciones B, 1998.

SUTTON, R.E. y WHEATLEY, K.F., "Teachers' emotions and teaching: A review of the literature and directions for future research", Educational psychology review, 15(4), 2003, pp.327-358.

VALLÉS-ARÁNDIGA, A., SICLE. Siendo inteligente con las emociones, Valencia, Promolibro, 1999. ZINS, J., PAYTON, J.W., WEISSBERG R.P., y O 'BRIEN, M.U. "Social and emotional learning and successful school performance”, en G. Matthews, M. Zeidner, \& R. D. Roberts (eds.), Emotional intelligence: Known's and unknowns, (pp. 376-395), New York, Oxford University Press, 2007. 